\title{
ON HIGHER TOPOLOGICAL HOCHSCHILD HOMOLOGY OF RINGS OF INTEGERS
}

\author{
BJØRN IAN DUNDAS, AYELET LINDENSTRAUSS, AND BIRGIT RICHTER
}

\begin{abstract}
We determine higher topological Hochschild homology of rings of integers in number fields with coefficients in suitable residue fields. We use the iterative description of higher THH for this and Postnikov arguments that allow us reduce the necessary computations to calculations in homological algebra, starting from the results of Bökstedt and Lindenstrauss-Madsen on (ordinary) topological Hochschild homology.
\end{abstract}

\section{INTRODUCTION}

Factorization homology provides a general framework for associating an invariant, $\int_{M} R$, to a topological $n$-dimensional manifold $M$ and an algebra $R$ over the Boardman-Vogt little $n$-cubes operad [BV73. For a comprehensive overview of factorization homology see AF15. Commutative algebras are algebras over the little $n$-cubes operad for all $n$. In [AF15, Proposition 5.1] it is shown that for commutative algebras $R$ the factorization homology, $\int_{M} R$, reduces to $R \otimes U(M)$ where $U(M)$ is the underlying topological space of $M$ and $(-) \otimes U(M)$ refers to the topological enrichment of the corresponding category.

In the setting of spectra, this shows that for a commutative ring spectrum $R$ the factorization homology with respect to an $n$-sphere, $\mathbb{S}^{n}$, can be expressed in classical terms as $R \otimes \mathbb{S}^{n}$ and this is what we call topological Hochschild homology of order $n$ of $R$.

We need a version with coefficients and we use simplicial sets as combinatorial models for topological spaces. It is shown in EKMM97, VII.3.2] that both enrichments (simplicial sets or topological spaces) give equivalent outcome. Topological Hochschild homology of a ring spectrum $R$ with coefficients in a module $N$ is obtained as a simplicial object where one uses the standard simplicial model of the 1-sphere, $S^{1}$, and glues $N$ to the basepoint of $S^{1}$ and $R$ to all other simplices in $S^{1}$. Topological Hochschild homology of order $n$ of a commutative ring spectrum $R$ with coefficients in $N, T H H^{[n]}(R, N)$, is the analogue of this where we use $S^{n}=\left(S^{1}\right)^{\wedge n}$ as a simplicial model of the $n$-sphere and glue again $N$ to the basepoint and $R$ to all other simplices of $S^{n}$. For a definition using the topological circle $\mathbb{S}^{1}$ see MSV97; for an approach preserving the epicyclic structure, see e.g., BCD10] or V18.

There are natural stabilization maps

$$
\pi_{*}\left(T H H^{[n]}(R, N)\right) \rightarrow \pi_{*+1}\left(T H H^{[n+1]}(R, N)\right)
$$

whose colimit gives the topological André-Quillen homology of $R$ with coefficients in $N$ as defined in Ba99.

Topological Hochschild homology, THH, of rings of integers in number fields is well-understood: Bökstedt Bö $\infty$ calculated THH of the integers and in [LM00, the general case is covered. The aim of this paper is the calculation of higher order topological Hochschild homology of rings of integers in number fields with coefficients in a suitable residue field.

If $N=R$, then we will abbreviate $T H H^{[n]}(R, R)$ as $T H H^{[n]}(R)$. If $A$ is a commutative discrete or simplicial ring and $M$ an $A$-module, we abuse notation and write $T H H^{[n]}(A)$ or $T H H^{[n]}(A, M)$ for $T H H^{[n]}(H A)$ or $T H H^{[n]}(H A, H M)$, respectively, where $H$ is the Eilenberg-Mac Lane spectrum functor.

John Rognes' redshift conjecture relates algebraic K-theory to chromatic phenomena: The stable homotopy category has a chromatic filtration and roughly the conjecture states that algebraic K-theory of a commutative ring spectrum of chromatic level $n$ is of chromatic level $n+1$, thus algebraic K-theory causes a chromatic redshift. See AR08, Conjecture 1.3] for a statement of the conjecture.

Replacing spheres by tori gives rise to torus homology and there is work by Brun, Carlsson, Douglas, Dundas, Rognes, Veen BCD10, CDD11, V18 and others on this. The hope is that, like algebraic K-theory, the homotopy fixed points with respect to the torus action of torus homology will also reveal red-shift 
phenomena. Positive results in that direction were obtained by Rognes and Veen [V18] and there is ongoing work by Ausoni and Dundas $\mathrm{AD} \infty$ extending these results to all chromatic layers. Both approaches use cellular decompositions of tori, and rely on concrete calculations for each cell, showing that higher topological Hochschild homology can be important for understanding chromatic phenomena.

Statement of results. In the following we give a brief description of our results and explain how one can use them together with local-to-global techniques and Bockstein spectral sequences in order to determine higher topological Hochschild homology of rings of integers in number fields.

We will phrase our results in terms of iterated Tor groups:

Definition 1.1. Let $k$ be a field. Let $B_{k}^{1}(x)$ be the polynomial algebra $P_{k}(x)$ on a generator $x$ in degree $2 m$. Inductively, we define the $k$-algebra $B_{k}^{n}(x)=\operatorname{Tor}_{*}^{B_{k}^{n-1}(x)}(k, k)$.

By Cartan [C54, $B_{k}^{2}(x)$ is the exterior algebra $\Lambda_{k}(\sigma x)$ on a generator $\sigma x$ in degree $2 m+1$; after that, we get a divided power algebra on a generator of degree $2 m+2$, and after that, the formulas become more complicated (but see BLPRZ15 for an illustration of what the $B_{k}^{n}(x)$ look like when $k$ is a finite field).

In the following, all tensors are over $\mathbb{F}_{p}$ unless otherwise explicitly marked. Our first result is:

Theorem 3.1 Let $n \geq 1$. Then $\operatorname{THH}_{*}^{[n]}\left(\mathbb{Z}, \mathbb{F}_{p}\right) \cong B_{\mathbb{F}_{p}}^{n}(x) \otimes B_{\mathbb{F}_{p}}^{n+1}(y)$ where $|x|=2 p$ and $|y|=2 p-2$.

In Remark 3.9 below we explain how to adapt our argument to show that

$$
T H H_{*}^{[n]}\left(\mathbb{F}_{p}\right) \cong B_{\mathbb{F}_{p}}^{n}(\mu)
$$

for a generator $\mu$ of degree two. This result was first obtained by Basterra and Mandell by other methods.

The calculation of $T H H_{*}^{[n]}\left(\mathbb{Z}, \mathbb{F}_{p}\right)$ along with a calculation of the Bockstein spectral sequence on it would give us $T H H_{*}^{[n]}(\mathbb{Z})$. We use this technique in DLR20 to determine $T H H_{*}^{[2]}\left(\mathbb{Z}_{(p)}\right)$ as a graded commutative ring (see [DLR20, Theorem 2.1]). An additive identification of $T H H_{*}^{[2]}(\mathbb{Z})$ is obtained in [K18, Theorem 1.6], using factorization homology and the identification of $H \mathbb{Z}$ as a Thom spectrum with an algebra structure over the little 2-cubes operad.

For a calculation of $T H H^{[n]}(A)$ for more general number rings $A$, observe first that for any $n$ and any commutative ring $A, T H H_{0}^{[n]}(A) \cong A$, since in the definition of $T H H^{[n]}(A)$, both $d_{0}$ and $d_{1}$ have to multiply all copies of $H A$ indexed on all the 1-simplices into the copy that sits over the basepoint, and so by the commutativity of $H A, d_{0}$ and $d_{1}$ induce the same maps on homology.

The Bökstedt spectral sequence for higher topological Hochschild homology (Proposition 7.2 in [BLPRZ15]) with rational coefficients is a spectral sequence with

$$
E_{*, *}^{2}=H H^{[n]}\left(H_{*}(H A ; \mathbb{Q})\right) \Rightarrow H_{*}\left(T H H^{[n]}(A) ; \mathbb{Q}\right) .
$$

Since $H_{*}(H A ; \mathbb{Q})$ consists just of $A \otimes \mathbb{Q}$ in dimension zero and since for a number ring $A, A \otimes \mathbb{Q}$ is étale over $\mathbb{Q}$, by Theorem 9.1(a) of [BLPRZ15, for $*>0, T H H_{*}^{[n]}(A)$ consists entirely of torsion.

However, since $A$ is a number ring, and hence a Dedekind domain, any finitely generated torsion module over it is a finite direct sum of modules $A / P_{i}^{k_{i}}$, with $P_{i}$ nonzero prime ideals and $k_{i} \geq 1$. For each such prime ideal $P_{i}$, there is a unique prime $p \in \mathbb{Z}$ for which $p A \subseteq P_{i}$, and if we consider $T H H^{[n]}(A)_{p}^{\wedge}$, its homotopy groups in positive dimensions will be all the modules $A / P_{i}^{k_{i}}$ where $p A \subseteq P_{i}$, and none of the others. The methods of Addendum 6.2 in [HM97, which show that for any number ring $A, T H H(A)_{p}^{\wedge} \simeq T H H\left(A_{p}^{\wedge}\right)_{p}^{\wedge}$, also show that for general $n \geq 1$,

$$
T H H^{[n]}(A)_{p}^{\wedge} \simeq T H H^{[n]}\left(A_{p}^{\wedge}\right)_{p}^{\wedge}
$$

So in order to understand the $P_{i}$ torsion in $T H H^{[n]}(A)$, we could see it instead in

$$
T H H^{[n]}\left(A_{p}^{\wedge}\right) \cong T H H^{[n]}\left(\prod_{P_{i} \text { prime, } p A \subseteq P_{i}} A_{P_{i}}^{\wedge}\right) \simeq \prod_{P_{i} \text { prime, } p A \subseteq P_{i}} T H H^{[n]}\left(A_{P_{i}}^{\wedge}\right) .
$$

Then, like calculating $T H H_{*}^{[n]}\left(\mathbb{Z}, \mathbb{F}_{p}\right)$ was an intermediate goal in the calculation of $T H H_{*}^{[n]}(\mathbb{Z})$, calculating $T H H_{*}^{[n]}\left(A_{P_{i}}^{\wedge},\left(A_{P_{i}}^{\wedge}\right) / P_{i}\right)$ is an intermediate goal in the calculation of $T H H_{*}^{[n]}\left(A_{P_{i}}^{\wedge}\right)$. 
We calculate the groups $T H H_{*}^{[n]}\left(A_{P_{i}}^{\wedge},\left(A_{P_{i}}^{\wedge}\right) / P_{i}\right)$ below, obtaining

Theorem 4.3 Let $A$ be the ring of integers in a number field, and let $P$ be a nonzero prime ideal in $A$. Denote the residue field $A / P$ by $\mathbb{F}_{q}$. Then

$$
T H H_{*}^{[n]}\left(A_{P}^{\wedge}, A / P\right) \cong B_{\mathbb{F}_{q}}^{n}\left(x_{P}\right) \otimes_{\mathbb{F}_{q}} B_{\mathbb{F}_{q}}^{n+1}\left(y_{P}\right)
$$

where

(i) $\left|x_{P}\right|=2$ and $\left|y_{P}\right|=0$ if $A$ is ramified over $\mathbb{Z}$ at $P$, and

(ii) $\left|x_{P}\right|=2 p$ and $\left|y_{P}\right|=2 p-2$, if $A$ is unramified over $\mathbb{Z}$ at $P$.

This gives the homotopy groups of

$$
T H H^{[n]}\left(A, A / P_{i}\right) \simeq T H H^{[n]}\left(A_{P_{i}}^{\wedge}\right) \wedge_{H\left(A_{P_{i}}\right)} H\left(A / P_{i}\right) .
$$

As in the $n=1$ case, multiplying $H \mathbb{Z}$ into the copy of $H\left(A_{P_{i}}^{\wedge}\right)$ over the basepoint shows that $T H H^{[n]}\left(A_{P_{i}}^{\wedge}\right)$ is a retract of $H \mathbb{Z} \wedge T H H^{[n]}\left(A_{P_{i}}^{\wedge}\right)$, and so additively, it is a product of Eilenberg-Mac Lane spectra. Any shifted copy $H\left(A / P_{i}^{k_{i}}\right), k_{i} \geq 1$, that we have in $T H H^{[n]}\left(A_{P_{i}}\right)$ will yield two correspondingly shifted copies of $H\left(A / P_{i}\right)$ (one with the same shift, one with that shift plus one) in $T H H^{[n]}\left(A_{P_{i}}^{\wedge}\right) \wedge_{H\left(A_{P_{i}}\right)} H\left(A / P_{i}\right)$. Again one can then read off the rank of the $P_{i}$-torsion from $T H H^{[n]}\left(A_{P_{i}}^{\wedge}, A / P_{i}\right)$, and to understand what the torsion actually is, one would need to look at Bockstein-type operators associated with multiplication by a uniformizer of $A_{P_{i}}^{\wedge}$.

\section{IDENTIFYING SQUARE ZERO EXTENSIONS}

Let $k$ be a commutative ring, and let $H k$ be the associated Eilenberg-Mac Lane commutative ring spectrum. We will show that there is exactly one homotopy type of augmented commutative $H k$-algebras $C$ with homotopy $\pi_{*} C \cong \Lambda_{k}(x)$ where $x$ is a generator in a given positive degree. That is, there is a chain of stable equivalences of augmented commutative $H k$-algebras $C \simeq H k \vee \Sigma^{m} H k$ where the $H k$-module $\Sigma^{m} H k$ is the $m$-fold suspension of $H k$ and $H k \vee \Sigma^{m} H k$ is the square-zero extension of $H k$ by $\Sigma^{m} H k$.

We learned of such a fact when $k=\mathbb{F}_{p}$ from Michael Mandell who proves it by means of topological André-Quillen homology and uses it in a program joint with Maria Basterra.

Proposition 2.1. Let $C$ be a commutative augmented $H k$-algebra and assume that there is an isomorphism of graded commutative $k$-algebras $\pi_{*} C \cong \Lambda_{k}(x)$ where $|x|=m>0$. Then there is a chain of stable equivalences of commutative augmented $H k$-algebras between $C$ and $H k \vee \Sigma^{m} H k$.

Proof. For concreteness, we formulate the proof in symmetric spectra. Let $S$ be the sphere spectrum, and $P_{S}$ and $P_{H k}$ the free commutative algebra functors (adjoint to the forgetful functor with values in $S$ or $H k$-modules). We may assume that $C$ is fibrant in the positive $H k$-model structure of Shipley [S04. Let $M$ be a positively $H k$-cofibrant resolution of $\Sigma^{m} H k$; for concreteness $M=H k \wedge F_{1}\left(\mathbb{S}^{m+1}\right)$, where $F_{1}$ is the adjoint to the evaluation on the first level. Represent $x$ by an $H k$-module morphism $M \rightarrow C$. Now,

$$
P_{H k}(M)=\bigvee_{n \geq 0}\left(M^{\wedge_{H k} n}\right)_{\Sigma_{n}} \cong H k \wedge P_{S}\left(F_{1}\left(\mathbb{S}^{m+1}\right)\right)
$$

is the free commutative $H k$-algebra on $M$. Since

$$
\left(F_{1}\left(\mathbb{S}^{m+1}\right)^{\wedge n}\right)_{\Sigma_{n}} \simeq\left(F_{1}\left(\mathbb{S}^{m+1}\right)^{\wedge n}\right)_{h \Sigma_{n}}
$$

is $(m n-1)$-connected and $m>0$, the induced map $f: P_{H k}(M) \rightarrow C$ is $2 m \geq(m+1)$-connected.

Taking the $m$ th Postnikov section $P_{m}$ of $P_{H k}(M)$ in the setting of commutative $H k$-algebra spectra (as done in the [EKMM97 setting in Ba99, §8]) gives a map of commutative $H k$-algebra spectra $P_{m} \rightarrow C$, which is an isomorphism on the non-zero homotopy groups in degree 0 and $m$. As both spectra are semistable [S $\infty$, 4.48], this map is a stable equivalence of symmetric spectra. Hence, $C$ and $P_{m}$ are of the same stable homotopy type, and repeating the argument with a fibrant model for $H k \vee \Sigma^{m} H k$ we get the promised chain of stable equivalences connecting $C$ and $H k \vee \Sigma^{m} H k$. 


\section{The Calculation of $T H H_{*}^{[n]}\left(\mathbb{Z}, \mathbb{F}_{p}\right)$}

Our goal in this section is to prove

Theorem 3.1. Let $n \geq 1$ and $p$ be any prime. Then

$$
T H H_{*}^{[n]}\left(\mathbb{Z}, \mathbb{F}_{p}\right) \cong B_{\mathbb{F}_{p}}^{n}(x) \otimes B_{\mathbb{F}_{p}}^{n+1}(y)
$$

where $|x|=2 p$ and $|y|=2 p-2$.

To this end we use the iterative description of $T H H^{[n]}$ : the $n$-sphere $\mathbb{S}^{n}$ can be decomposed into two hemispheres whose intersection is the equator, $\mathbb{S}^{n}=\mathbb{D}^{n} \cup_{\mathbb{S}^{n-1}} \mathbb{D}^{n}$. This decomposition yields V18 that

$$
T H H^{[n]}\left(\mathbb{Z}, \mathbb{F}_{p}\right) \simeq H \mathbb{F}_{p} \wedge_{T H H^{[n-1]}\left(\mathbb{Z}, \mathbb{F}_{p}\right)}^{L} H \mathbb{F}_{p}
$$

where $\wedge^{L}$ denotes the derived smash product.

Note that for any commutative ring spectrum $R, T H H(R)$ is a commutative $R$-algebra spectrum EKMM97. IX.2.2], so in particular, $T H H(\mathbb{Z})$ is a commutative $H \mathbb{Z}$-algebra spectrum. As $T H H\left(\mathbb{Z}, \mathbb{F}_{p}\right)=T H H(\mathbb{Z}) \wedge H \mathbb{Z}$ $H \mathbb{F}_{p}$, we have a commutative $H \mathbb{F}_{p}$-algebra structure on $T H H\left(\mathbb{Z}, \mathbb{F}_{p}\right)$ and the multiplication on $H \mathbb{Z}$ and the $H \mathbb{Z}$-module structure of $H \mathbb{F}_{p}$ give rise to a canonical augmentation from $T H H\left(\mathbb{Z}, \mathbb{F}_{p}\right)$ to $H \mathbb{F}_{p}$. Thus $T H H\left(\mathbb{Z}, \mathbb{F}_{p}\right)$ is a commutative augmented $H \mathbb{F}_{p}$-algebra spectrum.

Bökstedt $\underline{\mathrm{B} o ̈ \infty}$ calculated $\mathrm{THH}_{*}(\mathbb{Z})$ and his result gives the $n=1$ case of the theorem,

$$
T H H_{*}\left(\mathbb{Z}, \mathbb{F}_{p}\right) \cong \mathbb{F}_{p}\left[x_{2 p}\right] \otimes \Lambda\left(z_{2 p-1}\right)
$$

since $B_{\mathbb{F}_{p}}^{2}\left(y_{2 p-2}\right)$ is isomorphic to $\Lambda\left(z_{2 p-1}\right)$. We use the $(2 p-1)$ st Postnikov section of commutative augmented $H \mathbb{F}_{p}$-algebras to map $T H H_{*}\left(\mathbb{Z}, \mathbb{F}_{p}\right)$ to something which by Proposition 2.1 has to be weakly equivalent to $H \mathbb{F}_{p} \vee \Sigma^{2 p-1} H \mathbb{F}_{p}$. Then we consider the homotopy pushout diagram in the category of commutative augmented $H \mathbb{F}$-algebras

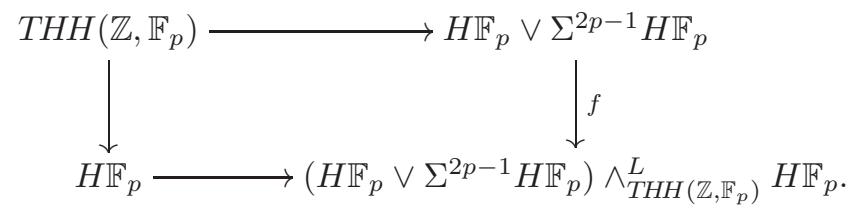

A bar spectral sequence argument tells us that the homotopy groups of $\left(H \mathbb{F}_{p} \vee \Sigma^{2 p-1} H \mathbb{F}_{p}\right) \wedge_{T H H\left(\mathbb{Z}, \mathbb{F}_{p}\right)}^{L} H \mathbb{F}_{p}$ are isomorphic to $\Lambda\left(y_{2 p+1}\right)$ and using Proposition 2.1 again we see that the homotopy pushout is a commutative augmented $H \mathbb{F}_{p}$-algebra which is weakly equivalent to $H \mathbb{F}_{p} \vee \Sigma^{2 p+1} H \mathbb{F}_{p}$.

The published version of this article unfortunately contains a mistake in the proof of Lemma 3.2 there. A variant of the proof which we present below was known to us at the time, but we sadly submitted a "simplification". The former Lemma 3.2 states (correctly) that the map

$$
f: H \mathbb{F}_{p} \vee \Sigma^{2 p-1} H \mathbb{F}_{p} \rightarrow H \mathbb{F}_{p} \vee \Sigma^{2 p+1} H \mathbb{F}_{p}
$$

of augmented commutative $H \mathbb{F}_{p}$-algebras from above factors in the homotopy category through the augmentation $H \mathbb{F}_{p} \vee \Sigma^{2 p-1} H \mathbb{F}_{p} \rightarrow H \mathbb{F}_{p}$, a fact which is a special case of the following result:

Lemma 3.2. Let $k$ be a discrete commutative ring, and let $a \neq b$ be two positive integers such that $b \leq 2 a$. Then any map in the category of commutative augmented Hk-algebras between the square-zero extensions

$$
H k \vee \Sigma^{a} H k \rightarrow H k \vee \Sigma^{b} H k
$$

factors in the homotopy category of commutative augmented Hk-algebras through the augmentation Hk $\vee$ $\Sigma^{a} H k \rightarrow H k$.

We use the notation of Basterra Ba99. For a commutative $\mathbb{S}$-algebra $R$, let $\mathcal{M}_{R}$ denote the category of $R$-modules, $\mathcal{N}_{R}$ denote the category of non-unital commutative $R$-algebras, and $\mathcal{C}_{R / R}$ denote the category of commutative augmented $R$-algebras. Consider the adjunctions

$$
\mathcal{N}_{R} \underset{Z}{\stackrel{Q}{\rightleftarrows}} \mathcal{M}_{R} \underset{U}{\stackrel{A}{\rightleftarrows}} \mathcal{N}_{R} \underset{I}{\stackrel{K}{\rightleftarrows}} \mathcal{C}_{R / R},
$$


where $U$ is the forgetful functor with left adjoint $A$, sending an $R$-module $M$ to the free non-unital commutative algebra on $M, U A M=\bigvee_{j>0} M^{\wedge_{R} j} / \Sigma_{j}$ with multiplication induced by concatenation; $Z$ is the square zero extension making an $R$-module $M$ into a nonunital commutative $R$-algebra $Z M$ using the trivial multiplication map sending everything to the basepoint, with left adjoint the indecomposables $Q$, sending a non-unital $R$-algebra to the pushout $Q C$ of $* \longleftarrow U\left(C \wedge_{R} C\right) \stackrel{\text { mult. }}{\longrightarrow} U C$; and, finally, $K$ is given by $K(M)=R \vee M$, with multiplication using $R$ 's multiplication, $M$ 's multiplication, and $M$ 's $R$-module structure, with right adjoint the augmentation ideal $I$, sending an augmented commutative $R$-algebra $B$ to the pullback $I(B)$ of $* \longrightarrow R \stackrel{\text { augm. }}{\longleftarrow} B$.

If $C$ is a $q$-cofibrant commutative $R$-algebra and $N \in \mathcal{M}_{R}$, then we get that the canonical equivalence $Z N \stackrel{\sim}{\rightarrow} I K Z N$ induces an equivalence

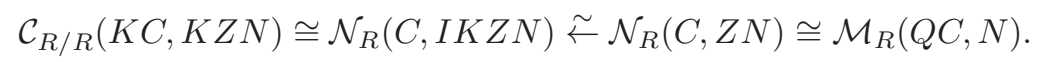

Proof of Lemma 3.2. In the special case of the lemma, letting $R$ be $H k$ and $C \stackrel{\sim}{\rightarrow} Z \Sigma^{a} R$ a $q$-cofibrant replacement in $\mathcal{N}_{R}$, we must show that the mapping space $\mathcal{M}_{R}\left(Q C, \Sigma^{b} R\right)$ is connected. Noting that $a \neq b$ implies that any map from $\Sigma^{a} R \simeq U C$ to $\Sigma^{b} R$ is nullhomotopic, we are done once we show the following more general Proposition 3.3 ,

In what follows $R$ is a connective $q$-cofibrant commutative $S$-algebra.

Proposition 3.3. Let $C \in \mathcal{N}_{R}$ have $\pi_{\ell} U C=0$ for $\ell<m$, let $N \in \mathcal{M}_{R}$ have $\pi_{\ell}(N)=0$ for $\ell>2 m$ and let $f: C \rightarrow Z N$ in the homotopy category ho $\mathcal{N}_{R}$ of non-unital commutative $R$-algebras be such that the underlying R-module map $U f: U C \rightarrow U Z N=N$ is nullhomotopic. Then $f$ is nullhomotopic.

In other words (using the isomorphism $\mathcal{N}_{R}(C, Z N) \cong \mathcal{M}_{R}(Q C, N)$ ), the map $U C \rightarrow Q C$ induces an injection ho $\mathcal{M}_{R}(Q C, N) \rightarrow h o \mathcal{M}_{R}(U C, N)$.

Letting $L C$ be the homotopy cofiber of the map $U C \rightarrow Q C$, we see that it is enough to prove that $\mathcal{M}_{R}(L C, N)$ is connected, which is immediate from the two following lemmas (with $X=L C$ and $n=2 m$ ).

Lemma 3.4. Let $X$ be a q-cofibrant $R$-module with $\pi_{\ell} X=0$ for $\ell \leq n$ and $N$ an $R$-module with $\pi_{\ell} N=0$ for $\ell>n$. Then $\mathcal{M}_{R}(X, N)$ is connected.

Proof. Since $R$ is connective, we can build $X$ by $R$-cells of dimension $\ell>n$ and $\mathcal{M}_{R}(X, N)$ is the limit of a tower of fibrations with connected fibers of the form $\mathcal{M}_{R}\left(\Sigma^{\ell} R, N\right) \simeq \Sigma^{-\ell} N$.

Lemma 3.5. Let $C$ be a q-cofibrant non-unital commutative $R$-algebra such that $\pi_{\ell} U C=0$ for $\ell<m$. Then the canonical map $U C \rightarrow Q C$ is $2 m$-connected.

Proof. Let $X \stackrel{\sim}{\rightarrow} U C$ be a $q$-cofibrant replacement in $\mathcal{M}_{R}$. Then, by induction on [Ba99, 5.3], the induced map $(U A)^{t} X \rightarrow(U A)^{t} U C \cong Q(A U)^{t+1} C$ is an equivalence and by [EKMM97, III.5.1] the counit $X \rightarrow(A U)^{t} X$ is $2 m$-connected, so that the canonical map $U C \rightarrow\left|[t] \mapsto Q(A U)^{t+1} C\right| \cong Q\left|[t] \mapsto(A U)^{t+1} C\right|$ is $2 m$ connected (where the simplicial structure is induced by the adjoint pair). Since, by [Ba99, 5.4], the map $Q\left|[t] \mapsto(A U)^{t+1} C\right| \rightarrow Q C$ is an equivalence, we are done.

We can therefore describe $T H H^{[2]}\left(\mathbb{Z}, \mathbb{F}_{p}\right)$ by the following iterated homotopy pushout diagram.

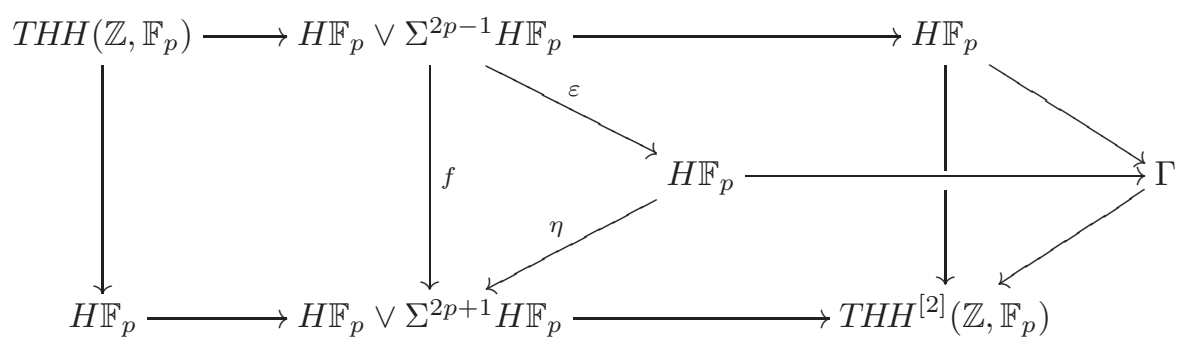

Here, $\Gamma$ denotes the homotopy pushout of the upper right subdiagram in the category of commutative $H \mathbb{F}_{p}$-algebras, and as above we get

$$
\Gamma=H \mathbb{F}_{p} \wedge_{H \mathbb{F}_{p} \vee \Sigma^{2 p-1} H \mathbb{F}_{p}}^{L} H \mathbb{F}_{p}
$$


We have again a Tor-spectral sequence converging to the homotopy groups of the spectrum $\Gamma$ with

$$
E_{*, *}^{2}=\operatorname{Tor}_{*, *}^{\Lambda\left(z_{2 p-1}\right)}\left(\mathbb{F}_{p}, \mathbb{F}_{p}\right)
$$

and hence $\pi_{*}(\Gamma)$ is isomorphic to a divided power algebra over $\mathbb{F}_{p}$ on a generator in degree $2 p, \Gamma\left(a_{2 p}\right)$. In the iterated homotopy pushout diagram all maps involved are maps of commutative $S$-algebras and thus we can identify $T H H^{[2]}\left(\mathbb{Z}, \mathbb{F}_{p}\right)$ as a commutative $H \mathbb{F}_{p}$-algebra as

$$
\begin{aligned}
T H H^{[2]}\left(\mathbb{Z}, \mathbb{F}_{p}\right) & \simeq\left(H \mathbb{F}_{p} \vee \Sigma^{2 p+1} H \mathbb{F}_{p}\right) \wedge_{H \mathbb{F}_{p} \vee \Sigma^{2 p-1} H \mathbb{F}_{p}}^{L} H \mathbb{F}_{p} \\
& \simeq\left(H \mathbb{F}_{p} \vee \Sigma^{2 p+1} H \mathbb{F}_{p}\right) \wedge_{H \mathbb{F}_{p}} \Gamma \\
& \cong \Gamma \vee \Sigma^{2 p+1} \Gamma .
\end{aligned}
$$

Thus, $T H H^{[2]}\left(\mathbb{Z}, \mathbb{F}_{p}\right)$ is equivalent to the bar construction

$$
B_{H \mathbb{F}_{p}}\left(H \mathbb{F}_{p}, H \mathbb{F}_{p} \vee \Sigma^{2 p-1} H \mathbb{F}_{p}, H \mathbb{F}_{p} \vee \Sigma^{2 p+1} H \mathbb{F}_{p}\right) .
$$

Its homotopy groups are

$$
T H H_{*}^{[2]}\left(\mathbb{Z}, \mathbb{F}_{p}\right) \cong \Gamma\left(a_{2 p}\right) \otimes \Lambda\left(y_{2 p+1}\right) .
$$

We use this to determine higher $T H H$ via iterated bar constructions. We know that $T H H^{[n+1]}\left(H \mathbb{Z}, H \mathbb{F}_{p}\right)$ is equivalent to the derived smash product

$$
H \mathbb{F}_{p} \wedge_{T H H^{[n]}\left(H \mathbb{Z}, H \mathbb{F}_{p}\right)}^{L} H \mathbb{F}_{p}
$$

whose homotopy groups are the ones of the bar construction

$$
B_{H \mathbb{F}_{p}}\left(H \mathbb{F}_{p}, T H H^{[n]}\left(H \mathbb{Z}, H \mathbb{F}_{p}\right), H \mathbb{F}_{p}\right)
$$

and iteratively, we can express $T H H^{[n]}\left(H \mathbb{Z}, H \mathbb{F}_{p}\right)$ again in terms of such a bar construction as long as $n$ is greater than two. For $n=2$ we know the answer by the above argument. For larger $n$ we can determine the homotopy groups of $T H H^{[n+1]}\left(H \mathbb{Z}, H \mathbb{F}_{p}\right)$ iteratively. Abbreviating $H \mathbb{F}_{p} \vee \Sigma^{2 p-1} H \mathbb{F}_{p}$ to $E(z)$ and $H \mathbb{F}_{p} \vee \Sigma^{2 p+1} H \mathbb{F}_{p}$ to $E(y)$ we define

$$
B^{(n)}:=B_{H \mathbb{F}_{p}}\left(H \mathbb{F}_{p}, \ldots, B_{H \mathbb{F}_{p}}\left(H \mathbb{F}_{p}, B_{H \mathbb{F}_{p}}\left(H \mathbb{F}_{p}, E(z), E(y)\right), H \mathbb{F}_{p}\right), \ldots, H \mathbb{F}_{p}\right)
$$

with $n-1$ pairs of outer terms of $H \mathbb{F}_{p}$. We denote by $\underline{E(y)}$ the constant simplicial $H \mathbb{F}_{p}$-algebra spectrum on $E(y)$.

Lemma 3.6. As n-simplicial commutative $H \mathbb{F}_{p}$-algebras

$$
B^{(n)} \simeq B_{H \mathbb{F}_{p}}^{(n)}\left(H \mathbb{F}_{p}, E(z), H \mathbb{F}_{p}\right) \wedge_{H \mathbb{F}_{p}} B_{H \mathbb{F}_{p}}^{(n-1)}\left(H \mathbb{F}_{p}, \underline{E(y)}, H \mathbb{F}_{p}\right)
$$

for all $n \geq 2$.

Proof. We show the claim directly for $n=2: B^{(2)}$ is

$$
B_{H \mathbb{F}_{p}}\left(H \mathbb{F}_{p}, B_{H \mathbb{F}_{p}}\left(H \mathbb{F}_{p}, E(z), E(y)\right), H \mathbb{F}_{p}\right) .
$$

As we know from Lemma 3.2 that the $E(z)$-module structure of $E(y)$ factors via the augmentation map through the $H \mathbb{F}_{p}$-module structure of $E(y)$, we get that $B_{H \mathbb{F}_{p}}\left(H \mathbb{F}_{p}, E(z), E(y)\right)$ can be split as an augmented simplicial commutative $H \mathbb{F}_{p}$-algebra as $B_{H \mathbb{F}_{p}}\left(H \mathbb{F}_{p}, E(z), H \mathbb{F}_{p}\right) \wedge_{H \mathbb{F}_{p}} \underline{E(y)}$ and thus we get a weak equivalence of bisimplicial commutative $H \mathbb{F}_{p}$-algebra spectra:

$$
\begin{aligned}
& B_{H \mathbb{F}_{p}}\left(H \mathbb{F}_{p}, B_{H \mathbb{F}_{p}}\left(H \mathbb{F}_{p}, E(z), E(y)\right), H \mathbb{F}_{p}\right) \\
\simeq & B_{H \mathbb{F}_{p}}\left(H \mathbb{F}_{p}, B_{H \mathbb{F}_{p}}\left(H \mathbb{F}_{p}, E(z), H \mathbb{F}_{p}\right) \wedge_{H \mathbb{F}_{p}} \underline{E(y)}, H \mathbb{F}_{p}\right) \\
\simeq & B_{H \mathbb{F}_{p}}\left(H \mathbb{F}_{p}, B_{H \mathbb{F}_{p}}\left(H \mathbb{F}_{p}, E(z), H \mathbb{F}_{p}\right), H \mathbb{F}_{p}\right) \wedge_{H \mathbb{F}_{p}} B_{H \mathbb{F}_{p}}\left(H \mathbb{F}_{p}, \underline{E(y)}, H \mathbb{F}_{p}\right) \\
= & B_{H \mathbb{F}_{p}}^{(2)}\left(H \mathbb{F}_{p}, E(z), H \mathbb{F}_{p}\right) \wedge_{H \mathbb{F}_{p}} B_{H \mathbb{F}_{p}}\left(H \mathbb{F}_{p}, \underline{E(y)}, H \mathbb{F}_{p}\right) .
\end{aligned}
$$

For the second weak equivalence in the chain above, we have used that the bar construction $B_{R}\left(R, X \wedge_{R} Y, R\right)$ of the smash product of two commutative simplicial $R$-algebra spectra $X$ and $Y$ is equivalent as a bisimplicial commutative $R$-algebra to $B_{R}(R, X, R) \wedge_{R} B(R, Y, R)$. 
By induction we assume that $n$ is bigger than 2 and that we know the result for all $k<n$. Then

$$
\begin{aligned}
B^{(n)}= & B_{H \mathbb{F}_{p}}\left(H \mathbb{F}_{p}, B^{(n-1)}, H \mathbb{F}_{p}\right) \\
\simeq & B_{H \mathbb{F}_{p}}\left(H \mathbb{F}_{p}, B_{H \mathbb{F}_{p}}^{(n-1)}\left(H \mathbb{F}_{p}, E(z), H \mathbb{F}_{p}\right)\right. \\
& \left.\wedge_{H \mathbb{F}_{p}} B_{H \mathbb{F}_{p}}^{(n-2)}\left(H \mathbb{F}_{p}, \underline{E(y)}, H \mathbb{F}_{p}\right), H \mathbb{F}_{p}\right) \\
\simeq & B_{H \mathbb{F}_{p}}\left(H \mathbb{F}_{p}, B_{H \mathbb{F}_{p}}^{(n-1)}\left(H \mathbb{F}_{p}, E(z), H \mathbb{F}_{p}\right), H \mathbb{F}_{p}\right) \\
& \wedge_{H \mathbb{F}_{p}} B_{H \mathbb{F}_{p}}\left(H \mathbb{F}_{p}, B_{H \mathbb{F}_{p}}^{(n-2)}\left(H \mathbb{F}_{p}, \underline{E(y)}, H \mathbb{F}_{p}\right), H \mathbb{F}_{p}\right) \\
= & B_{H \mathbb{F}_{p}}^{(n)}\left(H \mathbb{F}_{p}, E(z), H \mathbb{F}_{p}\right) \wedge_{H \mathbb{F}_{p}} B_{H \mathbb{F}_{p}}^{(n-1)}\left(H \mathbb{F}_{p}, \underline{E(y)}, H \mathbb{F}_{p}\right) .
\end{aligned}
$$

We view $T H H^{(n)}\left(H \mathbb{Z}, H \mathbb{F}_{p}\right)$ as a simplicial commutative $H \mathbb{F}_{p}$-algebra for all $n \geq 1$ and therefore describe $T H H^{(n+1)}\left(H \mathbb{Z}, H \mathbb{F}_{p}\right)$ as the diagonal of the bar construction $B_{H \mathbb{F}_{p}}\left(H \mathbb{F}_{p}, T H H^{[n]}\left(H \mathbb{Z}, H \mathbb{F}_{p}\right), H \mathbb{F}_{p}\right)$.

Corollary 3.7. We obtain, that

$$
\begin{aligned}
& T H H_{*}^{(n+1)}\left(H \mathbb{Z}, H \mathbb{F}_{p}\right) \\
\cong & \pi_{*} \operatorname{diag} B_{H \mathbb{F}_{p}}^{(n)}\left(H \mathbb{F}_{p}, E(z), H \mathbb{F}_{p}\right) \otimes_{\mathbb{F}_{p}} \pi_{*} \operatorname{diag} B_{H \mathbb{F}_{p}}^{(n-1)}\left(H \mathbb{F}_{p}, \underline{E(y)}, H \mathbb{F}_{p}\right) .
\end{aligned}
$$

For sake of definiteness in the following we will work in the category of symmetric spectra in simplicial sets, $\mathrm{Sp}^{\Sigma}$ [HSS00]. The Eilenberg-Mac Lane spectrum gives rise to a functor

$$
H: \mathcal{A} \rightarrow \mathrm{Sp}^{\Sigma}
$$

such that $H A(n)=\operatorname{diag}\left(A \otimes \tilde{\mathbb{Z}}\left(S^{n}\right)\right)$ where $S^{n}=\left(S^{1}\right)^{\wedge n}$ and $\tilde{\mathbb{Z}}(-)$ denotes the free abelian group generated by all non-basepoint elements. This functor is lax symmetric monoidal [S $\infty, 2.7,3.11$ ]. A square-zero extension $H \mathbb{F}_{p} \vee \Sigma^{n} H \mathbb{F}_{p}$ (for $n \geq 1$ ) can be modelled by $\mathbb{F}_{p}\left(\Delta_{n} / \partial \Delta_{n}\right)$ :

Lemma 3.8. There is a stable equivalence of commutative symmetric ring spectra $\psi: H \mathbb{F}_{p}\left(\Delta_{n} / \partial \Delta_{n}\right) \rightarrow$ $H \mathbb{F}_{p} \vee \Sigma^{n} H \mathbb{F}_{p}$.

Proof. There are two non-degenerate simplices in $\Delta_{n} / \partial \Delta_{n}$ : a zero-simplex $*$ corresponding to the unique basepoint and an $n$-simplex corresponding to the identity map id $\sin _{[3}$ on the set $[n]=\{0, \ldots, n\}$. We can represent any simplex in $\Delta_{n} / \partial \Delta_{n}$ as $s_{i_{\ell}} \circ \cdots \circ s_{i_{0}}(*)$ or $s_{i_{\ell}} \circ \cdots \circ s_{i_{0}}\left(\operatorname{id}_{[n]}\right)$. We define the map $\psi(m)_{\ell}$ from $H \mathbb{F}_{p}\left(\Delta_{n} / \partial \Delta_{n}\right)(m)_{\ell}=\mathbb{F}_{p}\left(\Delta_{n} / \partial \Delta_{n}\right)_{\ell} \otimes \tilde{\mathbb{Z}}\left(S_{\ell}^{m}\right)$ to $\left(H \mathbb{F}_{p} \vee \Sigma^{n} H \mathbb{F}_{p}\right)(m)_{\ell}=\mathbb{F}_{p} \otimes \tilde{\mathbb{Z}}\left(S_{\ell}^{m}\right) \vee \Delta_{n} / \partial \Delta_{n} \wedge \mathbb{F}_{p} \otimes \tilde{\mathbb{Z}}\left(S_{\ell}^{m}\right)$ on generators by setting

$$
\begin{aligned}
\psi\left(s_{i_{\ell}} \circ \cdots \circ s_{i_{0}}(*) \otimes x\right) & =1 \otimes x \in \mathbb{F}_{p} \otimes \tilde{\mathbb{Z}}\left(S_{\ell}^{m}\right) \\
\psi\left(s_{i_{\ell}} \circ \cdots \circ s_{i_{0}}\left(\operatorname{id}_{[n]}\right) \otimes x\right) & =\left[s_{i_{\ell}} \circ \cdots \circ s_{i_{0}}\left(\operatorname{id}_{[n]}\right), x\right] \in \Delta_{n} / \partial \Delta_{n} \wedge \mathbb{F}_{p} \otimes \tilde{\mathbb{Z}}\left(S_{\ell}^{m}\right)
\end{aligned}
$$

for $x \in S_{\ell}^{m}$ and by extending it in a bilinear manner. This map is well-defined and multiplicative. Both spectra have finite stable homotopy groups and are therefore semistable. It thus suffices to show that $\psi$ is a stable homotopy equivalence. The stable homotopy groups on both sides are exterior algebras on a generator in degree $n$ and $\psi$ induces the map on stable homotopy groups that sends 1 to 1 and maps the generator in degree $n$ to a degree $n$ generator.

We have a weak equivalence

$$
B_{H \mathbb{F}_{p}}\left(H \mathbb{F}_{p}, H A, H C\right) \rightarrow H\left(B_{\mathbb{F}_{p}}\left(\mathbb{F}_{p}, A, C\right)\right)
$$

for all simplicial $\mathbb{F}_{p}$-algebras $A$ and $C$. Let $N$ denote the normalization functor from simplicial $\mathbb{F}_{p^{-v e c t o r}}$ spaces to non-negatively graded chain complexes over $\mathbb{F}_{p}$. This is a lax symmetric monoidal functor, so it sends simplicial commutative $\mathbb{F}_{p}$-algebras to commutative differential graded $\mathbb{F}_{p}$-algebras. Note that we obtain isomorphisms of differential graded $\mathbb{F}_{p}$-algebras

$$
N\left(\mathbb{F}_{p}\left(\Delta_{2 p-1} / \partial \Delta_{2 p-1}\right)\right) \cong \Lambda_{\mathbb{F}_{p}}(z), \quad N\left(\mathbb{F}_{p}\left(\Delta_{2 p+1} / \partial \Delta_{2 p+1}\right)\right) \cong \Lambda_{\mathbb{F}_{p}}(y)
$$


because for positive $n, \Delta_{n} / \partial \Delta_{n}$ has only a non-degenerate zero cell and a non-degenerate $n$-cell. Note that $B_{\mathbb{F}_{p}}^{(n)}\left(\mathbb{F}_{p}, \mathbb{F}_{p}\left(\Delta_{2 p-1} / \partial \Delta_{2 p-1}\right), \mathbb{F}_{p}\right)$ is an $(n+1)$-fold simplicial commutative $\mathbb{F}_{p^{-}}$algebra. We can calculate the homotopy groups of its diagonal as the homology of the total complex associated to the bicomplex

$$
\begin{aligned}
& C_{r, s}=N_{r} \operatorname{diag}^{n} B_{\mathbb{F}_{p}}^{(n)}\left(\mathbb{F}_{p}, N_{s} \mathbb{F}_{p}\left(\Delta_{2 p-1} / \partial \Delta_{2 p-1}\right), \mathbb{F}_{p}\right) \\
& \cong N_{r} \operatorname{diag}^{n} B_{\mathbb{F}_{p}}^{(n)}\left(\mathbb{F}_{p}, \mathbb{F}_{p}\left(\Delta_{2 p-1} / \partial \Delta_{2 p-1}\right)_{s}, \mathbb{F}_{p}\right) \text {. }
\end{aligned}
$$

As the differential in $s$-direction is trivial the spectral sequence collapses at the $E^{2}$-term with total homology isomorphic to the given by the homology of the differential graded $n$-fold bar construction. These homology groups were calculated in [BLPRZ15] and we obtain that $\pi_{*} \operatorname{diag} B_{H \mathbb{F}_{p}}^{(n)}\left(H \mathbb{F}_{p}, E(z), H \mathbb{F}_{p}\right) \cong B_{\mathbb{F}_{p}}^{n+2}(y)$. Similarly, $\pi_{*} \operatorname{diag} B_{H \mathbb{F}_{p}}^{(n-1)}\left(H \mathbb{F}_{p}, \underline{E(y)}, H \mathbb{F}_{p}\right) \cong B_{\mathbb{F}_{p}}^{n+1}(x)$. This proves Theorem 3.1

Remark 3.9. Note that a similar argument as above shows that

$$
\mathrm{THH}_{*}^{[n]}\left(\mathbb{F}_{p}\right) \cong B_{\mathbb{F}_{p}}^{n}(\mu)
$$

for all $n \geq 1$ and all primes $p$. Here the degree of $\mu$ is two. For $n=1$ this calculation is due to Bökstedt Bö $\infty$. The only difference from the argument above is that the first step is slightly easier: in order to calculate $T_{H H}{ }^{[2]}\left(\mathbb{F}_{p}\right)$ we do not have to split off the Postnikov section - the bar spectral sequence immediately yields that $\mathrm{THH}_{*}^{[2]}\left(\mathbb{F}_{p}\right)$ is an exterior algebra on a class in degree 3. By Proposition 2.1 we know that we can model $T H H^{[2]}\left(\mathbb{F}_{p}\right)$ as $H \mathbb{F}_{p} \vee \Sigma^{3} H \mathbb{F}_{p}$. From there on the argument is completely analogous to the one above. Maria Basterra and Mike Mandell proved the isomorphism (3.9.0) for all $n$ and $p$ in 1999 using an $E_{\infty}$ bar construction argument. Partial results about higher $T H H$ of $\mathbb{F}_{p}$ were obtained by Rognes, Veen V18] and in BLPRZ15.

\section{THE NUMBER RING CASE}

The aim of this section is to prove Theorem 4.3, calculating the higher topological Hochschild homology of number rings with coefficients in the residue field. The calculation starts with the following observation.

Lemma 4.1. Let $B$ be a characteristic zero complete discrete valuation ring with residue field $\mathbb{F}_{q}$ of characteristic $p>0$ and let $P$ denote the ideal consisting of all elements with positive valuation in $B$. Then

$$
H H_{1}^{\mathbb{Z}_{p}}(B, B / P) \cong \begin{cases}B / P, & \text { if } B \text { is ramified over } \mathbb{Z}_{p} \text { at } P, \\ 0, & \text { otherwise. }\end{cases}
$$

Proof. By Proposition 12 in Chapter 3 of $[\mathbf{S 7 9}], B$ is generated over $\mathbb{Z}_{p}$ by a single element, $B=\mathbb{Z}_{p}[x] /(f(x))$ for some monic polynomial $f$. By a well-known calculation which can be traced back to [T57], for any ring $R$ and monic polynomial $f(x)$ over it, $H H_{1}^{R}(R[x] /(f(x))) \cong R[x] /\left(f(x), f^{\prime}(x)\right)$. By Corollary 2 of Chapter 3 of [S79], the ideal $\left(f^{\prime}(x)\right)$ in $\mathbb{Z}_{p}[x] /(f(x))$ is equal to the different ideal $\mathcal{D}_{B / \mathbb{Z}_{p}}$, so the result for $B$ over $\mathbb{Z}_{p}$ becomes

$$
H H_{1}^{\mathbb{Z}_{p}}(B) \cong B / \mathcal{D}_{B / \mathbb{Z}_{p}}
$$

Since $B$ is commutative, we also know that $H H_{0}^{\mathbb{Z}_{p}}(B) \cong B$ is free over $B$, hence $\operatorname{Tor}_{s}^{B}\left(H H_{0}^{\mathbb{Z}_{p}}(B), B / P\right)=0$ for $s>0$. If we tensor the Hochschild complex of $B$ over $B$ with $B / P$, then we get by the universal coefficient theorem, that

$$
H H_{1}^{\mathbb{Z}_{p}}(B, B / P) \cong H H_{1}^{\mathbb{Z}_{p}}(B) \otimes_{B} B / P \cong B / \mathcal{D}_{B / \mathbb{Z}_{p}} \otimes_{B} B / P \cong B /\left(\mathcal{D}_{B / \mathbb{Z}_{p}}, P\right)
$$

If $\mathcal{D}_{B / \mathbb{Z}_{p}} \subseteq P$, this is just $B / P$, but if not, by the maximality of $P$ in $B$ we get that $B /\left(\mathcal{D}_{B / \mathbb{Z}_{p}}, P\right) \cong 0$. Theorem 1 in Chapter 3 of [S79] says that an extension $B$ of $\mathbb{Z}_{p}$ is ramified at an ideal $P$ of $B$ if and only if $P$ divides the different ideal $\mathcal{D}_{B / \mathbb{Z}_{p}}$.

From this we establish the one-dimensional (ordinary topological Hochschild homology) case of Theorem 4.3, which is closely related to Theorem 4.4 in [LM00, and in fact in the unramified case is exactly Theorem 4.4 (i) there (since in the unramified case $P=p A$ for a rational prime $p$ ). The symbol $x_{i}$ in the statement indicates a generator of degree $i$. 
Proposition 4.2. Let $A$ be the ring of integers in a number field, and let $P$ be a nonzero prime ideal in $A$. Denote the residue field $A / P$ by $\mathbb{F}_{q}$.

(i) If $A$ is ramified over $\mathbb{Z}$ at $P, T H H_{*}^{[1]}\left(A_{P}^{\wedge}, \mathbb{F}_{q}\right) \cong \mathbb{F}_{q}\left[x_{2}\right] \otimes_{\mathbb{F}_{q}} \Lambda_{\mathbb{F}_{q}}\left[x_{1}\right]$.

(ii) If $A$ is unramified over $\mathbb{Z}$ at $P, \operatorname{THH}_{*}^{[1]}\left(A_{P}^{\wedge}, \mathbb{F}_{q}\right) \cong \mathbb{F}_{q}\left[x_{2 p}\right] \otimes_{\mathbb{F}_{q}} \Lambda_{\mathbb{F}_{q}}\left[x_{2 p-1}\right]$.

Proof. We set $B=A_{P}^{\wedge}$, to get a ring that satisfies the conditions of Lemma 4.1. We now use $P$ to denote the ideal in $B$ obtained as $P B$ for the ideal $P$ of $A$. We use Morten Brun's spectral sequence [Br00, p. 30] from Theorem 3.3 in LM00 for the map $B \rightarrow B / P$. This gives a multiplicative spectral sequence

$$
E_{r, s}^{2}=T H H_{r}\left(B / P, \operatorname{Tor}_{s}^{B}(B / P, B / P)\right) \Rightarrow T H H_{r+s}(B, B / P) .
$$

Since $P$ is a principal ideal in $B$, generated by any uniformizer $\pi$, the resolution

$$
0 \longrightarrow B \stackrel{\cdot \pi}{\longrightarrow} B \longrightarrow B / P \longrightarrow 0
$$

shows that $\operatorname{Tor}_{*}^{B}(B / P, B / P) \cong \Lambda_{\mathbb{F}_{q}}\left(\tau_{1}\right)$ for a 1-dimensional generator $\tau_{1}$, where $B / P=\left(A_{P}^{\wedge}\right) / P \cong A / P=\mathbb{F}_{q}$.

Bökstedt showed in $\underline{B} \ddot{\infty}$, that $T H H_{*}\left(\mathbb{F}_{p}\right) \cong \mathbb{F}_{p}\left[u_{2}\right]$, and since $H H_{*}\left(\mathbb{F}_{q}\right)$ consists only of $\mathbb{F}_{q}$ in dimension zero, the spectral sequence of Theorem 2.2 in [L00]

$$
E_{r, s}^{2}=H H_{r}^{\mathbb{F}_{p}}\left(\mathbb{F}_{q}, T H H_{s}\left(\mathbb{F}_{p} ; \mathbb{F}_{q}\right)\right) \Rightarrow T H H_{r+s}\left(\mathbb{F}_{q}\right)
$$

consists only of $\mathbb{F}_{q} \otimes \mathbb{F}_{p}\left[u_{2}\right]$ in the zeroth row, we get that

$$
T H H_{*}\left(\mathbb{F}_{q}\right) \cong \mathbb{F}_{q}\left[u_{2}\right] .
$$

Thus Brun's spectral sequence takes the form

$$
E_{*, 0}^{2} \cong \mathbb{F}_{q}\left[u_{2}\right], \quad E_{*, 1}^{2} \cong \tau_{1} \cdot \mathbb{F}_{q}\left[u_{2}\right] .
$$

From Lemma 4.1 and the fact that Hochschild and topological Hochschild homology agree in degree 1, we know that we end up with nothing in total degree 1 if $B$ is unramified over $\mathbb{Z}_{p}$, and with a copy of $\mathbb{F}_{q}$ if $B$ is ramified. So we get

$$
d^{2}\left(u_{2}\right)= \begin{cases}0, & \text { if } B \text { is ramified over } \mathbb{Z}_{p} \text { at } P, \\ (\text { unit }) \cdot \tau_{1}, & \text { otherwise. }\end{cases}
$$

In the ramified case we already know that $d^{2}$ vanishes on 1 and $\tau_{1}$, since there is nothing these elements could hit. Therefore, we get that $d^{2}=0$. As $d^{2}$ is the last differential that could be nontrivial, $E_{*, *}^{\infty} \cong E_{*, *}^{2} \cong$ $\Lambda_{\mathbb{F}_{q}}\left(\tau_{1}\right) \otimes_{\mathbb{F}_{q}} \mathbb{F}_{q}\left[u_{2}\right]$, and since this is the multiplication with the fewest relations possible that could be defined on a graded-commutative algebra with this linear structure, extensions cannot give any other multiplicative structure and we get

$$
T H H_{*}(B, B / P) \cong \Lambda_{\mathbb{F}_{q}}\left(\tau_{1}\right) \otimes_{\mathbb{F}_{q}} \mathbb{F}_{q}\left[u_{2}\right] .
$$

In the unramified case, the knowledge what $d^{2}$ does on the generators shows us that $d^{2}\left(u_{2}^{a}\right)=($ unit $) \cdot \tau_{1}$. $u_{2}^{a-1}$ when $p$ does not divide $a$, but $d^{2}\left(u_{2}^{p k}\right)=0$ and nothing hits the elements $\tau_{1} \cdot u_{2}^{p k-1}$. Again $d^{2}$ is the last differential that could be nonzero so $E_{*, *}^{\infty} \cong E_{*, *}^{3} \cong \Lambda_{\mathbb{F}_{q}}\left(\tau_{1} \cdot u_{2}^{p-1}\right) \otimes_{\mathbb{F}_{q}} \mathbb{F}_{q}\left[u_{2}^{p}\right]$, and since this is again a multiplication with the fewest relations possible that could be defined on a graded-commutative algebra with this linear structure, extensions cannot give any other multiplicative structure and we get

$$
T H H_{*}(B, B / P) \cong \Lambda_{\mathbb{F}_{q}}\left(\tau_{1} \cdot u_{2}^{p-1}\right) \otimes_{\mathbb{F}_{q}} \mathbb{F}_{q}\left[u_{2}^{p}\right] .
$$

Theorem 4.3. Let $A$ be the ring of integers in a number field, and let $P$ be a nonzero prime ideal in $A$. Denote the residue field $A / P$ by $\mathbb{F}_{q}$.

Then

$$
T H H_{*}^{[n]}\left(A_{P}^{\wedge}, \mathbb{F}_{q}\right) \cong B_{\mathbb{F}_{q}}^{n}\left(x_{P}\right) \otimes_{\mathbb{F}_{q}} B_{\mathbb{F}_{q}}^{n+1}\left(y_{P}\right)
$$

where

(i) $\left|x_{P}\right|=2$ and $\left|y_{P}\right|=0$ if $A$ is ramified over $\mathbb{Z}$ at $P$, and

(ii) $\left|x_{P}\right|=2 p$ and $\left|y_{P}\right|=2 p-2$, if $A$ is unramified over $\mathbb{Z}$ at $P$. 
Proof. The $n=1$ case is true by Proposition 4.2, with $x=x_{2}$ and $y$ zero-dimensional (so that $B_{\mathbb{F}_{q}}^{1}(y) \cong$ $\left.\Lambda_{\mathbb{F}_{q}}\left(x_{1}\right)\right)$ in the ramified case, and with $x=x_{2 p}$ and $y$ of dimension $2 p-2$ (so that $\left.B_{\mathbb{F}_{q}}^{1}(y) \cong \Lambda_{\mathbb{F}_{q}}\left(x_{2 p-1}\right)\right)$ in the unramified case.

The rest of the proof proceeds by exact analogy to the calculation of $T H H_{*}^{[n]}\left(\mathbb{Z}, \mathbb{F}_{p}\right)$.

Note 4.4. The ramified case Theorem 4.3 (i) can actually be proven quite algebraically by noting that for an arbitrary flat ring $A$ and $A$-bimodule $M$, the linearization map $\operatorname{THH}(A, M) \rightarrow H\left(H H^{\mathbb{Z}}(A, M)\right)$ is 3connected so that the first Postnikov sections of THH $(A, M)$ and $H\left(H H^{\mathbb{Z}}(A, M)\right)$ agree. As a matter of fact, when $A$ is a $\mathbb{Z}_{(p)}$-algebra, Bökstedt's calculation of the topological Hochschild homology of the integers gives that Theorem 2.3 of $\left[\mathrm{L} 00\right.$ implies that this can be improved to saying that $T H H(A, M) \rightarrow H\left(H H^{\mathbb{Z}}(A, M)\right)$ is $(2 p-1)$-connected. This means that the Postnikov section involved in the crucial step moving from THH to to the algebraic $\mathrm{THH}^{[2]}$ coincides with that of Hochschild homology. This was how we originally established the calculation in the ramified case.

Note 4.5. The unramified case, Theorem 4.3](ii), could have also been deduced from Theorem 3.1] by showing that

$$
T H H_{*}^{[n]}\left(A_{P}^{\wedge}, A / P\right) \cong T H H_{*}^{[n]}\left(\mathbb{Z}_{p}, \mathbb{F}_{p}\right) \otimes \mathbb{F}_{q} \quad\left(\text { where } \mathbb{F}_{q}=A / P\right)
$$

as an augmented $\mathbb{F}_{q}$-algebra, where the augmentation on the right-hand side comes from the augmentation of $T H H_{*}^{[n]}\left(\mathbb{Z}, \mathbb{F}_{p}\right)$ over $\mathbb{F}_{p}$, tensored with the identity of $\mathbb{F}_{q}$. This is true for $n=1$ by Theorem 4.4 (i) of LM00, and then we proceed inductively, using the decomposition from (3.1.0). This yields in this case a decomposition

$$
T H H^{[n+1]}\left(A_{P}^{\wedge}, \mathbb{F}_{q}\right) \simeq H \mathbb{F}_{q} \wedge_{T H H^{[n]}\left(A_{P}^{\wedge}, \mathbb{F}_{q}\right)}^{L} H \mathbb{F}_{q} .
$$

and a multiplicative spectral sequence

$$
\operatorname{Tor}_{*, *}^{T H H_{*}^{[n]}}\left(A_{P}^{\wedge}, \mathbb{F}_{q}\right)\left(\mathbb{F}_{q}, \mathbb{F}_{q}\right) \Rightarrow T H H_{*}^{[n+1]}\left(A_{P}^{\wedge}, \mathbb{F}_{q}\right),
$$

which can be rewritten by the inductive hypothesis as

$$
\begin{aligned}
& \operatorname{Tor}_{*, *}^{T H H_{*}^{[n]}}\left(\mathbb{Z}_{p}, \mathbb{F}_{p}\right) \otimes \mathbb{F}_{q}\left(\mathbb{F}_{p} \otimes \mathbb{F}_{q}, \mathbb{F}_{p} \otimes \mathbb{F}_{q}\right) \\
& \cong \operatorname{Tor}_{*, *}^{T H H_{*}^{[n]}}\left(\mathbb{Z}_{p}, \mathbb{F}_{p}\right)\left(\mathbb{F}_{p}, \mathbb{F}_{p}\right) \otimes \operatorname{Tor}_{*}^{\mathbb{F}_{q}}\left(\mathbb{F}_{q}, \mathbb{F}_{q}\right) \\
& \cong \operatorname{Tor}_{*, *}^{T H H_{*}^{[n]}}\left(\mathbb{Z}, \mathbb{F}_{p}\right)\left(\mathbb{F}_{p}, \mathbb{F}_{p}\right) \otimes \mathbb{F}_{q},
\end{aligned}
$$

where the first factor is the image of the $E^{2}$-term of the spectral sequence calculating $T H H_{*}^{[n+1]}\left(\mathbb{Z}, \mathbb{F}_{p}\right)$ and the second term is in $E_{0,0}^{2}$ and therefore can cause no nontrivial differentials or multiplicative extensions. This splitting is a splitting of algebras and the augmentation is that of the first factor tensored with the identity of the second.

\section{ACKNOWLEDGEMENTS}

This material is based upon work supported by the National Science Foundation under Grant No. 0932078000 while the authors were in residence at the Mathematical Sciences Research Institute in Berkeley California, during the Spring 2014 program on Algebraic Topology.

We are grateful to Mike Mandell for providing the crucial hint that there is just one homotopy type of augmented commutative $H \mathbb{F}_{p}$-algebras with homotopy groups $\Lambda_{\mathbb{F}_{p}}(x)$.

\section{REFERENCES}

$[\mathrm{AD} \infty] \quad$ Christian Ausoni and Bjørn Ian Dundas, Detecting red-shift via torus homology, in preparation.

[AR08] Christian Ausoni and John Rognes, The chromatic red-shift in algebraic K-theory, in: Guido's Book of Conjectures, Monographie de L'Enseignement Mathématique 40 (2008), 13-15.

[AF15] David Ayala and John Francis, Factorization homology of topological manifolds, J. Topol. 8 (2015), no. 4, 10451084.

[BGR08] Andrew Baker, Helen Gilmour, and Philipp Reinhard, Topological André-Quillen homology for cellular commutative $S$-algebras, Abh. Math. Semin. Univ. Hambg. 78 (2008), no. 1, 27-50.

[Ba99] Maria Basterra, André-Quillen cohomology of commutative S-algebras, J. Pure Appl. Algebra 144 (1999), no. 2, 111-143. 
[BaM05] Maria Basterra and Michael A. Mandell, Homology and cohomology of E $\infty_{\infty}$-ring spectra, Math. Z. 249 (2005), no. 4, 903-944.

[BV73] J. Michael Boardman and Rainer M. Vogt, Homotopy invariant algebraic structures on topological spaces, Lecture Notes in Mathematics, Vol. 347. Springer-Verlag, Berlin-New York (1973), x+257pp.

[BLPRZ15] Irina Bobkova, Ayelet Lindenstrauss, Kate Poirier, Birgit Richter, and Inna Zakharevich, On the higher topological Hochschild homology of $\mathbb{F}_{p}$ and commutative $\mathbb{F}_{p}$-group algebras, in: Women in Topology: Collaborations in Homotopy Theory. Cont. Math. 641, AMS (2015), 97-122.

[Bö $\infty] \quad$ Marcel Bökstedt, The topological Hochschild homology of $\mathbb{Z}$ and of $\mathbb{Z} / p \mathbb{Z}$, preprint.

[Br00] Morten Brun, Topological Hochschild homology of $\mathbb{Z} / p^{n}$, J. Pure Appl. Algebra 148 (2000), no. 1, 29-76.

[BCD10] Morten Brun, Gunnar Carlsson, and Bjørn Ian Dundas, Covering homology, Adv. Math. 225 (2010), $3166-3213$.

[C54] Henri Cartan, Détermination des algèbres $H_{*}\left(\pi, n ; Z_{p}\right)$ et $H^{*}\left(\pi, n ; Z_{p}\right)$, p premier impair, Exp. No. 9, 10 p., Séminaire Henri Cartan, 7 no. 1, 1954-1955, Algèbre d'Eilenberg-Maclane et homotopie (available at http://www.numdam.org/).

[CDD11] Gunnar Carlsson, Christopher L. Douglas, and Bjørn Ian Dundas Higher topological cyclic homology and the Segal conjecture for tori, Adv. Math. 226 (2011), no. 2, 1823-1874.

[DLR20] Bjørn Ian Dundas, Ayelet Lindenstrauss, and Birgit Richter, Towards an understanding of ramified extensions of structured ring spectra, Mathematical Proceedings of the Cambridge Philosophical Society 168 (3) (2020), 435-454.

[EKMM97] Anthony D. Elmendorf, Igor Kriz, Michael A. Mandell, and J. Peter May, Rings, modules, and algebras in stable homotopy theory, With an appendix by M. Cole. Mathematical Surveys and Monographs 47. American Mathematical Society, Providence, RI (1997), xii+249pp.

[HM97] Lars Hesselholt and Ib Madsen, On the K-theory of finite algebras over Witt vectors of perfect fields, Topology 36 (1997), no. 1, 29-101.

[HSS00] Mark Hovey, Brooke Shipley, and Jeff Smith, Symmetric spectra, J. Amer. Math. Soc. 13 (2000), no. 1, 149-208.

[K18] Inbar Klang, The factorization theory of Thom spectra and twisted nonabelian Poincaré duality, Algebr. Geom. Topol. 18 (2018), no. 5, 2541-2592.

[L00] Ayelet Lindenstrauss, A relative spectral sequence for topological Hochschild homology of spectra, J. Pure Appl. Algebra 148 (2000), no. 1, 77-88.

[LM00] Ayelet Lindenstrauss, and Ib Madsen, Topological Hochschild homology of number rings, Trans. Amer. Math. Soc. 352 (2000), no. 5, 2179-2204.

[MSV97] James E. McClure, Roland Schwänzl, and Rainer Vogt, $T H H(R) \cong R \otimes \mathbb{S}^{1}$ for $E_{\infty}$ ring spectra, J. Pure Appl. Algebra 121 (1997), no. 2, 137-159.

$[\mathrm{S} \infty]$ Stefan Schwede, An untitled book project about symmetric spectra, available at http://www.math.uni-bonn.de/people/schwede/SymSpec.pdf

[S79] Jean-Pierre Serre, Local Fields, Graduate Texts in Mathematics 67, Springer Verlag, New York-Berlin (1979), viii+241 pp.

[S04] Brooke Shipley, A convenient model category for commutative ring spectra, In Homotopy theory: relations with algebraic geometry, group cohomology, and algebraic $K$-theory, Contemp. Math. 346 (2004), 473-483.

[T57] John Tate, Homology of Noetherian rings and local rings, Illinois J. Math. 1 (1957), 14-27.

[V18] Torleif Veen, Detecting periodic elements in higher topological Hochschild homology, Geom. Topol. 22 (2018), no. $2,693-756$.

Department of Mathematics, University of Bergen, Postboks 7803, 5020 Bergen, Norway

Email address: dundas@math.uib.no

Department of Mathematics, Indiana University, Bloomington IN 47405, USA

Email address: alindens@indiana.edu

Fachbereich Mathematik, Universität Hamburg, Bundesstrasse 55, 20146 Hamburg, Germany

Email address: birgit.richter@uni-hamburg.de 